\title{
Magnetic field simulation of a thermal conductivity measurement instrument for magnetorheological fluid
}

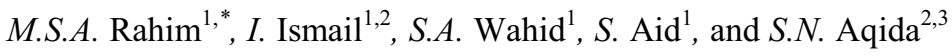 \\ ${ }^{1}$ Faculty of Manufacturing Engineering, Universiti Malaysia Pahang, 26600 Pekan, Pahang, Malaysia \\ ${ }^{2}$ Automotive Engineering Centre, Universiti Malaysia Pahang, 26600 Pekan, Pahang, Malaysia \\ ${ }^{3}$ Faculty of Mechanical Engineering, Universiti Malaysia Pahang, 26600 Pekan, Pahang, Malaysia
}

\begin{abstract}
Technological advancements in thermal systems demand an innovative heat dissipation technology. Magnetorheological (MR) fluid has a huge potential to solve the problem. However, characterising thermal conductivity of the materials in magnetic fields required tailored instruments. This paper presents a concept design of the MR fluids thermal conductivity measurement instrument. The developed instrument was designed to be able to measure thermal conductivity in both parallel and perpendicular orientations with magnetic field. Magnetic fields distribution of the proposed concept design was analysed using finite element method for magnetics. Design modification then conducted to improve the magnetic fields strength. Findings of this study showed that gap thickness played a significant factor in determining the optimal design. Simulated magnetic fields strength at both parallel and perpendicular orientations were found identical, yet varied in distributions.
\end{abstract}

\section{Introduction}

Magnetorheological (MR) fluid is a suspension that possesses alterable rheological properties in magnetic field presence. Due to this advantage, MR fluid is beneficial to be applied in active and semi-active devices [1]. At high temperature environment, shear thinning and decreasing shear stress of MR fluid devices could occur that eventually resulted in loss of efficiency. One of the ways to anticipate this is to improve on thermal conductivity property of MR fluid.

Thermal conductivity is greatly enhanced when MR fluid is subjected to magnetic field [2]. The finding was experimentally done by Yildirim and Genc [3] in which the thermal conductivity of MR fluid increased along with increasing magnetic field. This behaviour is attributed to the formation of chain-like structures by the magnetic particles when subjected to magnetic field, which provide high conductivity heat transfer paths [4]. Additionally, thermal conductivity of MR fluid is found to be anisotropic under the influence of magnetic field. Reinecke et al. [5] found that thermal conductivity of MR fluid increased by almost $100 \%$ when thermal gradient is parallel with magnetic field.

\footnotetext{
* Corresponding author: sallehrahim@icloud.com
} 
There are several ways to measure thermal conductivity property of a suspension. The most widely used is transient hot-wire (THW) method. THW method has the capacity to eliminate error due to natural convection besides getting faster results compared to other methods [6]. Very recently, Khdher et al. [7] adopted this method to obtain thermal conductivity of bio glycol based Al2O3 nanofluids. Gavili et al. [8] have used THW to measure thermal conductivity of water based ferrofluids. Cheng et al. [9] used THW at different MR fluid concentrations in the absence of magnetic field.

However, the THW method is unable to measure anisotropic thermal conductivity of MR fluid. This limitation has pushed researchers to adopt different methods. Cha et al. [10] has developed a transient hot-square instrument that enabled direct measurements of thermal conductivity in the direction parallel to external magnetic field. Krichler and Odenbach [11] used hot-plate technique where specimen is positioned between two plates. The idea is for both parallel and perpendicular arrangements of thermal gradient and magnetic field are possible.

This paper is aimed to obtain the optimal design for a thermal conductivity measurement instrument. The instrument will be able to measure thermal conductivity of MR fluid with the presence of magnetic field. It is also capable to measure the anisotropic thermal conductivity of MR fluid at parallel and perpendicular directions to magnetic field. Geometry parameters of the instrument are being considered. The influence of each parameter for several designs will be measured by magnetic flux density. Analysis and design optimization are carried out by using Finite Element Method Magnetics (FEMM) software.

\section{Design methodology}

The method used to measure thermal conductivity is based on a guarded hot plate technique and followed ISO 8301 standard. Usually this technique is applied to solid body measurements, for which the specimen is positioned in between two plates. The upper plate is heated, and the lower plate maps the temperature increase. During each measurement, the heater located in the upper plate is subjected to a current pulse and the resulting change in its electrical resistance and hence temperature is monitored as a function of time. However, modification of the system has been done to accommodate the magnets for magnetic field induction. This modification is beyond requirement of the standard. This method has also been applied in previous research [3, 10, 11].

In this design, a MR fluid sample is filled inside a PMMA container. A plate heater (upper plate) is used as the heat source and is embedded inside the cap. Heat sink (lower plate) acted as the base for the instrument and to release heat from the top. A pocket is made each at the top of the cap and at the bottom of the heat sink as slots for the permanent magnets. The attraction of the magnets will then generate a vertically stable magnetic field through the PMMA. The slots at the side of the PMMA are also created for the magnets to produce horizontal magnetic field. Several thermocouples are installed around the PMMA wall to monitor the change of temperature. The schematic diagram of the thermal conductivity measurement instrument is shown in Figure 1.

The research began by identifying geometry parameters of the instrument. The parameters included were PMMA diameter, PMMA height and gap thickness between magnets and sample. They were then categorized to three different levels; low, medium and high. Geometry parameters of the proposed design were selected at medium level. Such design was chosen after considering that the design geometries are suitable for ease of manufacturing and assembly. However, consideration of low and high levels of geometry parameters are also significant as to observe the influence and magnitude of each parameter. The summary of geometry parameters is as shown in Table 1. 


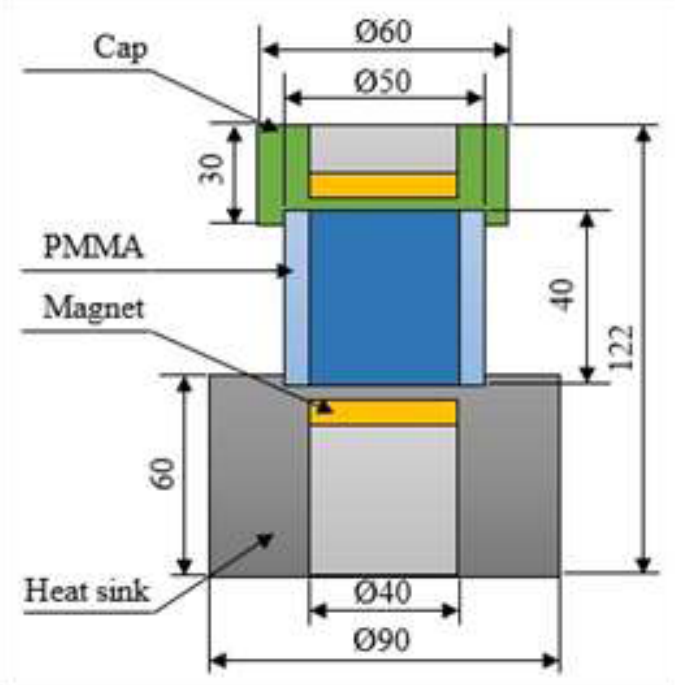

Fig. 1. Schematic diagram of thermal conductivity measurement instrument.

The diameter and height of the cylindrical PMMA were set to be equal in geometry regardless of their level. These were designed in such a way that the difference of thermal conductivity between parallel and perpendicular directions of magnetic field can be observed. The gap is what separates the magnet with the sample. On the other hand, the plate heater inside the cap indicates that thermal gradient is only unidirectional (vertical).

Table 1. Geometry parameters of thermal conductivity measurement instrument.

\begin{tabular}{|c|c|c|c|}
\hline Geometry parameters & Low & Medium & High \\
\hline PMMA diameter, $\mathbf{m m}$ & 30 & 40 & 50 \\
\hline PMMA height, $\mathbf{m m}$ & 30 & 40 & 50 \\
\hline Gap thickness, $\mathbf{m m}$ & 5 & 10 & 15 \\
\hline
\end{tabular}

Nevertheless, the main property to be focused on in this research was the magnetic flux density. Magnetic flux density was obtained from the two permanent magnets which were located and arranged either vertically or horizontally. The magnet thickness was $10 \mathrm{~mm}$. Magnetic field from the magnets will create heat conduction paths across the instrument through the formation of chains in the MR fluid. Chain formation process in an MR fluid only acquires several milliseconds to complete after the magnetic field is applied [12]. The sample used in the simulation was MRF-132DG, a commercial MR fluid. The minimum magnetic flux density required to develop chain formation for MRF-132DG is approximately $0.0597 \mathrm{~T}$ [13].

\subsection{Material of instrument}

Due to many desirable properties, polymethyl methacrylate (PMMA) has been selected as the cylindrical wall material. It possesses high mechanical strength, high Young's modulus and low elongation at break. PMMA is also considered among the hardest thermoplastics and is highly scratch resistant. Meanwhile, thermal stability of standard PMMA is $65^{\circ} \mathrm{C}$. Heat stabilised types can withstand temperatures of up to $100^{\circ} \mathrm{C}$. The typical properties of PMMA material (Cyro Industries, USA) can be found in Table 2. 
Table 2. Typical properties of polymethyl methacrylate (PMMA).

\begin{tabular}{|c|c|}
\hline Properties & Value \\
\hline Hardness, Rockwell & M80-M100 \\
\hline Tensile strength & $55-76 \mathrm{MPa}$ \\
\hline Tensile modulus & $2413-3447 \mathrm{MPa}$ \\
\hline Max. operating temperature & $65-93^{\circ} \mathrm{C}$ \\
\hline Thermal conductivity & $0.56 \mathrm{~W} / \mathrm{m} . \mathrm{K}$ \\
\hline Melting point & $130-140^{\circ} \mathrm{C}$ \\
\hline
\end{tabular}

Polytetrafluoroethylene (PTFE) offers high chemical resistance, low and high temperature capability, resistance to weathering, low friction, electrical and thermal insulation, and "slipperiness". PTFE's mechanical properties are low compared to other plastics, but its properties remain at a useful level over a wide temperature range of $-73^{\circ} \mathrm{C}$ to $204^{\circ} \mathrm{C}$. It has excellent thermal and electrical insulation properties and a low coefficient of friction. Hence, PTFE is very suitable as the cap material. The typical properties of PTFE material (DuPont, USA) are as depicted in Table 3.

Table 3. Typical properties of polytetrafluoroethylene (PTFE).

\begin{tabular}{|c|c|}
\hline Properties & Value \\
\hline Hardness, Shore D & D 50 \\
\hline Tensile strength & $26-27 \mathrm{MPa}$ \\
\hline Tensile modulus & $550 \mathrm{MPa}$ \\
\hline Max. operating temperature & $260^{\circ} \mathrm{C}$ \\
\hline Thermal conductivity & $0.25 \mathrm{~W} / \mathrm{m} \cdot \mathrm{K}$ \\
\hline Melting point & $335^{\circ} \mathrm{C}$ \\
\hline
\end{tabular}

The material selected for heat sink was aluminium. Possessing high thermal conductivity, aluminium allows heat to pass through it swiftly. Meanwhile, the disk shaped permanent magnets used were NdFeB (Neodymium-Iron-Boron) N52 grade. N52 is the highest material grade with residual flux density of $1.43 \mathrm{~T}$ and coercivity of $891.3 \mathrm{kA} / \mathrm{m}$. The maximum energy product for N52 is 52 MGOe.

\subsection{Finite element method for magnetics}

The simulation was a magnetostatic problem in which the fields are time-invariant. In this case, the magnetic field intensity, $H$ and magnetic flux density, $B$ must obey:

$$
\begin{gathered}
\nabla \times H=J \\
\nabla \cdot B=0
\end{gathered}
$$

Finite Element Method Magnetics (FEMM) software goes about finding a field that satisfies Eqs. (1) and (2) via a magnetic vector potential approach [14]. Adopting the $B$ and $H$ relationship equation and flux density equation, (1) can be rewritten as:

$$
\nabla \times\left(\frac{1}{\mu(B)} \nabla \times A\right)=J
$$


$\nabla$ is the divergence of a vector field,

$\mathrm{H}$ is the magnetic field intensity,

$\mathrm{J}$ is the electric current density,

$\mathrm{B}$ is the magnetic flux density,

$\mu$ is the magnetic permeability,

$\mathrm{A}$ is the vector potential.

The thermal conductivity measurement instrument was designed in FEMM software and was analyzed as a 2-D planar model. The proposed design was with vertical magnet arrangement, PMMA geometries of $\varnothing 40 \times 40 \mathrm{~mm}$, and $10 \mathrm{~mm}$ gap thickness at each side. Materials were defined accordingly for each design parts. The gap material at the top was set to PTFE while at the bottom was set to Aluminum 6061-T6. This difference can be neglected as both are linear materials (relative magnetic permeability, $\mu_{\mathrm{r}}=1$ ) and have no coercivity. FEMM generated 8890 nodes and 17418 elements for the proposed design. The mesh shape was set to triangular while the mesh size had an average of $0.5 \mathrm{~mm}$ size. The boundary condition type was a mix of Robin or Neumann. For this simulation, the mixed boundary condition was treated as open boundary problem.

\section{Magnetic field simulation}

The magnetic field simulation results are given in Figure 2 and Figure 3. The low level of geometry parameters is represented by Figure 2 (a) and Figure 3 (a) while the high level is represented by Figure 2 (b) and Figure 3 (b). Warmer colours correspond to higher flux densities while solid black lines indicate magnetic field lines. The magnitude of flux density was at the maximum at the edge of each disk shaped magnets. It is also observed that the magnetic field lines were crossing through the PMMA. The closest point inside PMMA to the magnet had the highest magnitude of flux density while the centre point inside PMMA had the lowest magnitude of flux density. Hence, the MR fluid samples filled inside the PMMA containers for vertical and horizontal magnet arrangements were experiencing magnetization.

Since the magnets produced uniform magnetic fields, attractive or repulsive fieldparticle interactions do not exist due to the lack of a magnetic field gradient [15]. The particle-particle interactions in the MR fluid become dominant. The imposed field induces magnetic dipoles and as a result, mutual particle interactions occur. The magnetic particles in the MR fluid attract each other when aligned end to end. Due to the attractive forces, chain-like structures are developed .

The forming of chain-like structures from magnetic particles in the MR fluid created heat conduction paths. Since magnet arrangements in Figure 2 (a) and Figure 2 (b) were vertical with plate heater at the top, the conduction paths will be parallel to the thermal gradient. On the other hand, horizontal magnet arrangements in Figure 3 (a) and Figure 3 (b) will be perpendicular to the thermal gradient. Hence, the anisotropic thermal conductivity of MR fluid under the influence of magnetic field can be observed.

\section{Design modifications}

In vertical magnet arrangement, the magnitude of flux density at the centre point of PMMA in Figure 2 (a) and Figure 2 (b) were $0.166 \mathrm{~T}$ and $0.115 \mathrm{~T}$, respectively. Comparing with the proposed design which has $\varnothing 40 \times 40 \mathrm{~mm}$ of PMMA and $10 \mathrm{~mm}$ gap thickness, the magnitude was recorded at $0.143 \mathrm{~T}$. Visually and mathematically, all designs produced 
higher flux densities than required $(0.0597 \mathrm{~T})$ to develop chain formations of magnetic particles [13].

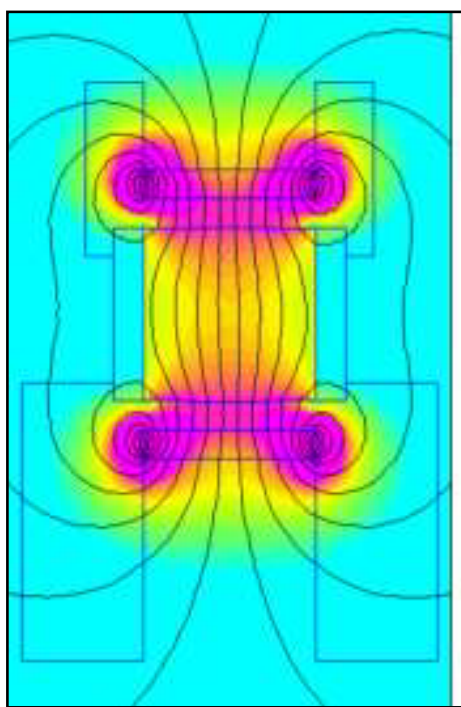

(a)

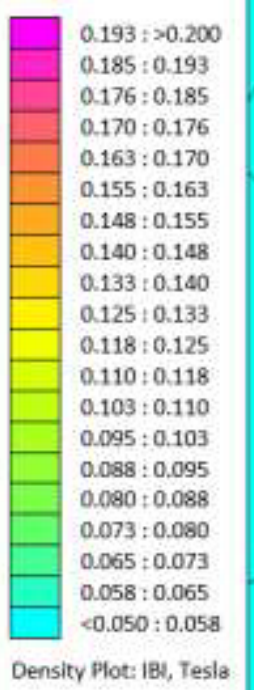

Density Plot: iBu, Tesla

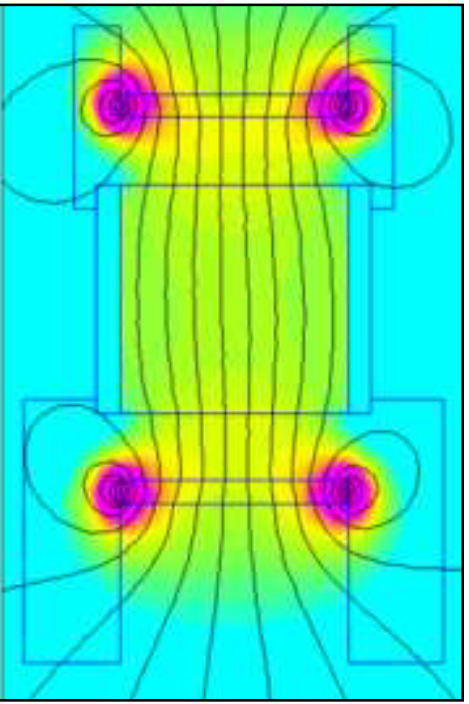

(b)

Fig. 2. Magnetic flux density for vertical magnet arrangement of (a) Ø30 x $30 \mathrm{~mm}$ of PMMA and (b) $\varnothing 50 \times 50 \mathrm{~mm}$ of PMMA at $5 \mathrm{~mm}$ and $15 \mathrm{~mm}$ magnet gap; respectively.

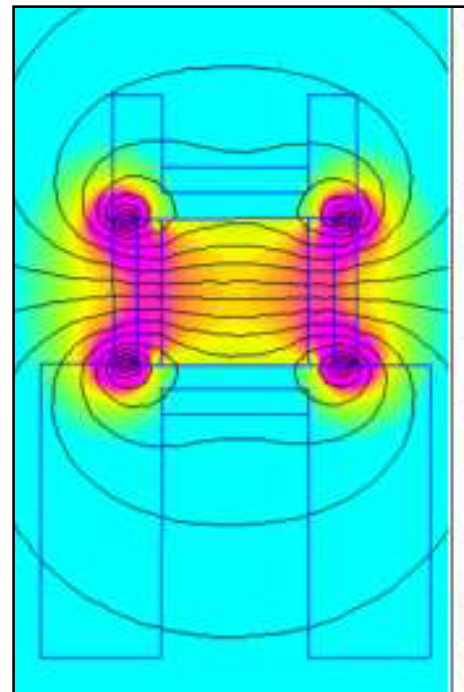

(a)

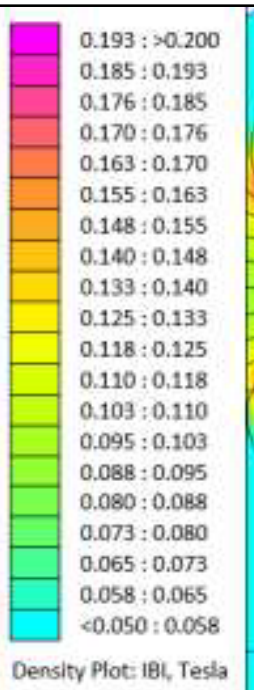

Density Plot: 181, Tesla

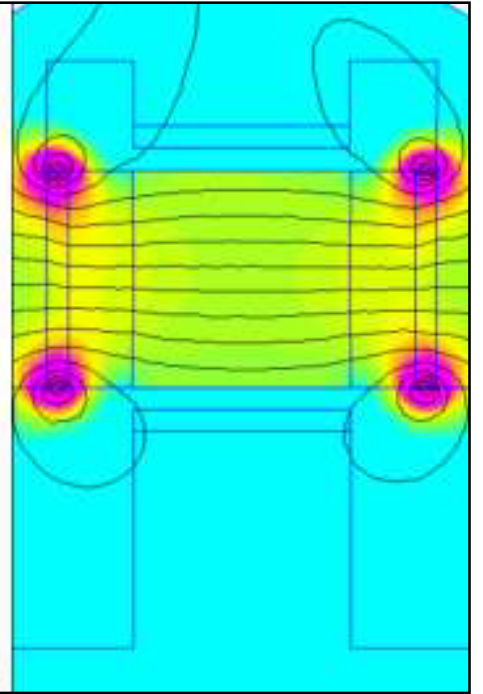

(b)

Fig. 3. Magnetic flux density for horizontal magnet arrangement of (a) Ø30 x $30 \mathrm{~mm}$ of PMMA and (b) Ø50 x $50 \mathrm{~mm}$ of PMMA at $5 \mathrm{~mm}$ and $15 \mathrm{~mm}$ magnet gap; respectively.

Figure 4 highlights on the effect of PMMA geometries to magnetic flux density at 10 $\mathrm{mm}$ gap thickness. By varying PMMA heights, there is not much difference to the magnitude of flux density. A Ø30 × $30 \mathrm{~mm}$ of PMMA resulted in $0.159 \mathrm{~T}$ while a $\varnothing 30 \times 50$ $\mathrm{mm}$ of PMMA achieved at $0.155 \mathrm{~T}$. At $\varnothing 40 \mathrm{~mm}$ and $\varnothing 50 \mathrm{~mm}$, the difference is almost negligible where changes of flux density were only $0.001 \mathrm{~T}$ across all heights. The standard 
deviations for $30 \mathrm{~mm}, 40 \mathrm{~mm}$ and $50 \mathrm{~mm}$ of PMMA heights are $0.002 \mathrm{~T}, 0.001 \mathrm{~T}$ and 0.001 $\mathrm{T}$, respectively.

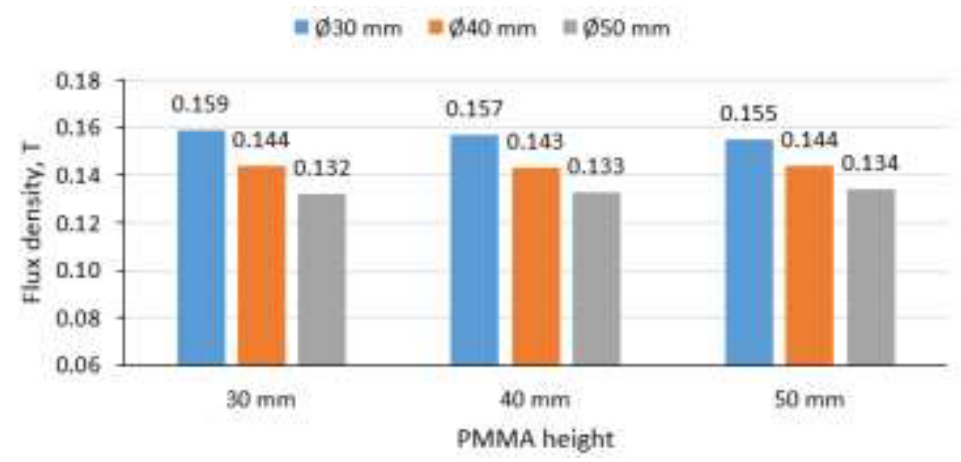

Fig. 4. Effect of different PMMA diameter and height (gap thickness $=10 \mathrm{~mm}$ ).

A minor effect is also observed by changing the PMMA diameter. The trend shows that magnetic flux density decreases as PMMA diameter increases across any given height. The largest flux density difference can be seen at $30 \mathrm{~mm}$ PMMA height. Flux density was 0.159 $\mathrm{T}$ at $\varnothing 30 \mathrm{~mm}$ but decreased to $0.132 \mathrm{~T}$ at $\varnothing 50 \mathrm{~mm}$. The standard deviations for $\varnothing 30 \mathrm{~mm}$, $\varnothing 40 \mathrm{~mm}$ and $\varnothing 50 \mathrm{~mm}$ are $0.014 \mathrm{~T}, 0.012 \mathrm{~T}$ and $0.011 \mathrm{~T}$, respectively. Hence, it is presumed that the distance between two magnets and diameter of the magnet (due to the increase of PMMA diameter) are insignificant due to oversupply of magnetic field strength from the strong magnets.

Meanwhile, the effect of gap thickness at constant PMMA geometry is as depicted in Figure 5. It is clearly found that $5 \mathrm{~mm}$ of gap thickness gave the highest magnitude of flux density. At $\varnothing 30 \times 30 \mathrm{~mm}$, the flux density was captured at $0.213 \mathrm{~T}$ for $5 \mathrm{~mm}$ gap thickness compared to $0.114 \mathrm{~T}$ for $15 \mathrm{~mm}$ gap. The impact is great as there is minimal boundary between magnets and sample. Then again, the declination also applies to $\varnothing 40 \times 40 \mathrm{~mm}$ and $\varnothing 50 \times 50 \mathrm{~mm}$ as gap thickness increases but has much smaller influence. At $15 \mathrm{~mm}$ gap thickness, there is hardly any change of flux density though PMMA geometries are different.

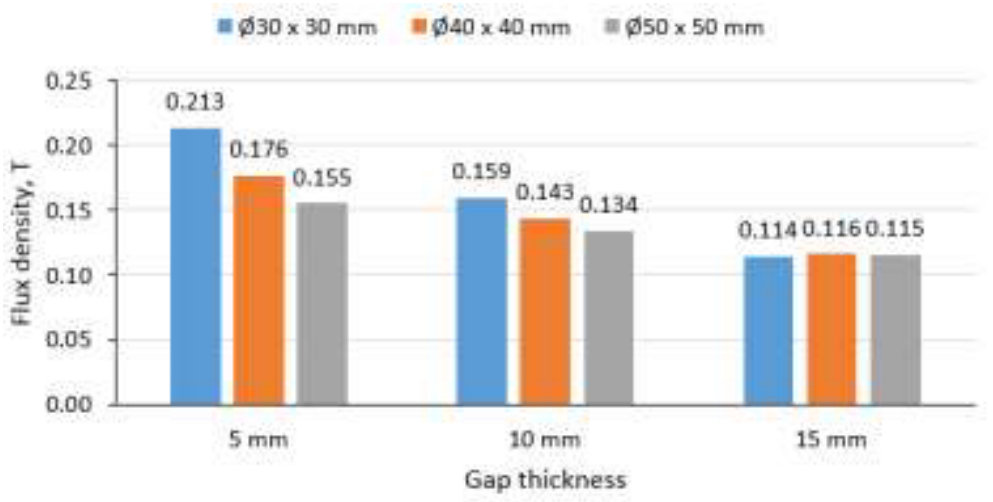

Fig. 5. Effect of gap thickness at constant PMMA diameter and height.

On the other hand, the magnitude of flux density for horizontal magnet arrangement was $0.215 \mathrm{~T}$ in Figure 3 (a) and 0.113 in Figure 3 (b). The proposed design was recorded at $0.147 \mathrm{~T}$. As similar to vertical magnet arrangement, all designs are able to develop chain formations of magnetic particles. 
Figure 6 shows on the effect of PMMA geometry to flux density with $10 \mathrm{~mm}$ gap thickness. At different PMMA width, changes of flux density were not much. The flux density was $0.153 \mathrm{~T}$ at $\varnothing 30 \times 30 \mathrm{~mm}$ and decreased to $0.151 \mathrm{~T}$ at $\varnothing 50 \times 30 \mathrm{~mm}$. This indicates that the PMMA width shows little influence on flux density. It also shows that the horizontal magnet arrangement is having similar effect with the vertical magnet arrangement. This is because the location of magnets is simply changed from vertical to horizontal and hence the height of PMMA at vertical becomes the width of PMMA at horizontal. The PMMA height also shows minimal impact but with greater influence than PMMA width. Flux density of $0.153 \mathrm{~T}$ was recorded at $\varnothing 30 \times 30 \mathrm{~mm}$ and reduced to 0.136 $\mathrm{T}$ for $\varnothing 30 \times 50 \mathrm{~mm}$ at the same width. The standard deviations for PMMA height of 30 $\mathrm{mm}, 40 \mathrm{~mm}$ and $50 \mathrm{~mm}$ are $0.009,0.008$ and 0.007 ; respectively.

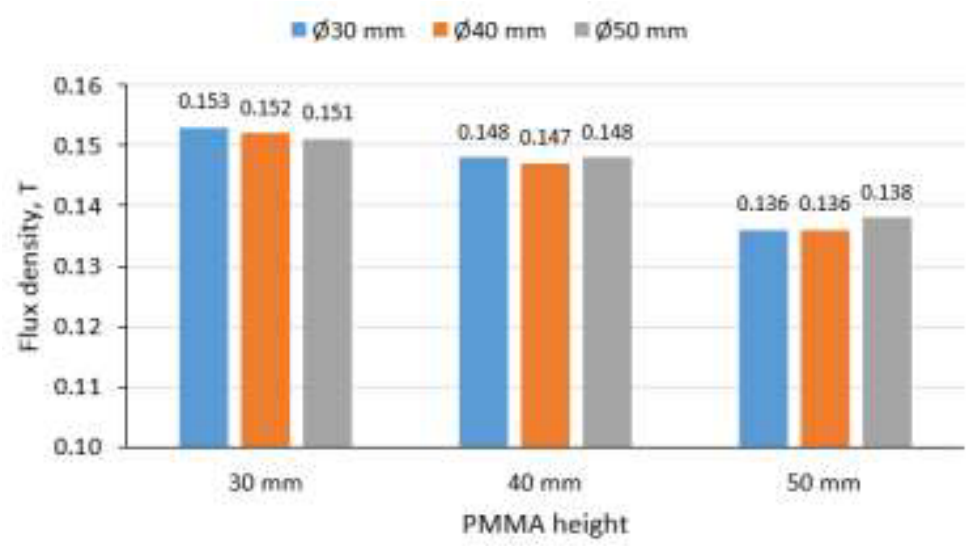

Fig. 6. Effect of different PMMA diameter and height (gap thickness $=10 \mathrm{~mm})$.

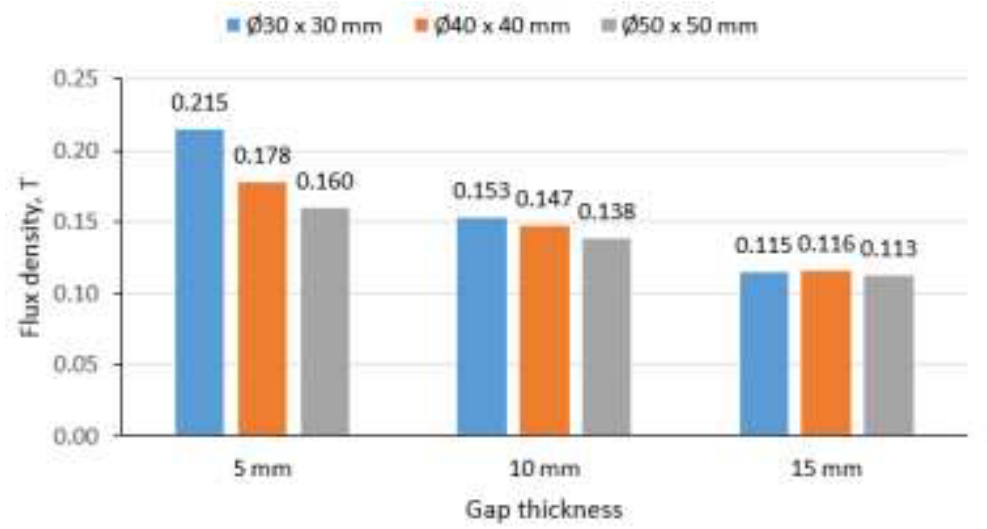

Fig. 7. Effect of gap thickness at constant PMMA diameter and height.

The effect of gap thickness with the same PMMA geometry is observed in Figure 7. As similar to vertical arrangement, the outcome is also larger at $5 \mathrm{~mm}$ gap thickness in horizontal magnet arrangement. Flux density was recorded at $0.215 \mathrm{~T}$ at $5 \mathrm{~mm}$ gap but reduced to $0.115 \mathrm{~T}$ at $15 \mathrm{~mm}$ gap. The changes are smaller as PMMA geometry is bigger due to reduced influence of magnetic field. 


\section{Conclusions}

This work has suggested several design considerations for a thermal conductivity measurement instrument. Three parameters are being looked into namely PMMA diameter, PMMA height and gap thickness. By utilizing the FEMM software, simulation results were produced and magnetic flux density for all designs have been achieved. It is clearly found that gap thickness played a significant factor in determining the optimal design. For both vertical and horizontal magnet arrangements, the highest magnetic flux densities were produced with gap thickness at $5 \mathrm{~mm}$. Meanwhile, the geometries of PMMA container indicated a minimal flux density change at all parameter levels. For vertical magnet arrangement, the low level $(\varnothing 30 \mathrm{~mm})$ of PMMA diameter resulted in higher flux density regardless of the PMMA height. This is a complete opposite to horizontal magnet arrangement in which the PMMA height at low level $(30 \mathrm{~mm})$ is more dominant, irrespective of PMMA diameter. Hence, the optimal design for the thermal conductivity measurement instrument is by having PMMA geometries at medium level with $5 \mathrm{~mm}$ of gap thickness.

The authors are gratefully acknowledged Universiti Malaysia Pahang (UMP) for providing facilities and financial support.

\section{References}

1. I. Ismail, S.A. Mazlan, Z. Hairi, A.G. Olabi, Jpn. J. Appl. Phys. 51, 1 (2012)

2. M.S.A. Rahim, I. Ismail, Mater. Sci. Eng. 100, 1 (2015)

3. G. Yildirim, S. Genc, Smart Mater. Struct. 22, 1 (2013)

4. S.I. Mistik, T. Shah, R.L. Hadimani, E. Siores, J. Intel. Mat. Syst. Str. 23, 1277 (2012)

5. B.N. Reinecke, J.W. Shan, K.K. Suabedissen, A.S. Cherkasova, J. Appl. Phys. 104, 1 (2008)

6. G. Paul, M. Chopkar, I. Manna, P.K. Das, Renew. Sust. Energ. Rev. 14, 1913 (2010)

7. A.M. Khdher, N.A.C. Sidik, W.A.W. Hamzah, R. Mamat, Int. Commun. Heat. Mass. 73, 75 (2016)

8. A. Gavili, F. Zabihi, T.D. Isfahani, J. Sabbaghzadeh, Exp. Therm. Fluid. Sci. 41, 94 (2012)

9. H. Cheng, W. Hong, C. Liu, J. Chin. Adv. Mat. S. 2, 130 (2014)

10. G. Cha, Y.S. Ju, L.A. Ahuré, N.M. Wereley, J. Appl. Phys. 107, 1 (2010)

11. M. Krichler, S. Odenbach, J. Magn. Magn. Mater. 326, 85 (2013)

12. J. de Vicente, D.J. Klingenberg, R.H. Alvarez, Soft Matter 7, 3701 (2011)

13. N. Wiltsie, M. Lanzetta, K. Iagnemma, TePRA, 91 (2012)

14. D. Meeker, Finite Element Method Magnetics Version 4.2 User's Manual (2015)

15. M. Zrínyi, Colloid Polym Sci 278, 98 (2000) 